\title{
LAS FILOSOFÍAS DE LA CIENCIA DE THOMAS KUHN: DEL CICLO REVOLUCIONARIO AL ÁRBOL EVOLUTIVO
}

\author{
THOMAS KUHN'S PHILOSOPHIES OF SCIENCE: \\ FROM REVOLUTIONARY CYCLE TO EVOLUTIONARY TREE
}

\author{
James A. Marcum*
}

\begin{abstract}
Resumen
En este artículo exploro la transición en el pensamiento de Kuhn hacia una filosofía evolutiva de la ciencia a partir de una filosofía histórica de la ciencia. Esta primera filosofía de la ciencia de Kuhn se ha representado repetidas veces como un proceso cíclico en el que una antigua ciencia normal da lugar a un nueva ciencia normal a través de una revolución científica o un cambio de paradigma. Más tarde en su carrera Kuhn rechaza la filosofía histórica de la ciencia y se vuelca hacia una filosofía evolutiva. En lugar de entender el progreso científico como un levantamiento revolucionario, lo concibe ahora como la creciente especialización de las disciplinas científicas. De ahí que, en contraste con un ciclo revolucionario, un árbol evolutivo representa mejor la filosofía de la ciencia madura de Kuhn acerca de como progresa de la ciencia.
\end{abstract}

Palabras clave: árbol evolutivo, inconmensurabilidad, Kuhn, ciclo revolucionario, simbiogénesis.

\footnotetext{
Abstract

In this paper, I explore Kuhn's turn from a historical philosophy of science to an evolutionary one. The former philosophy of science is often represented as a cycle in which an older normal science gives way to a newer one through a scientific revolution or paradigm shift. Later in his career, Kuhn rejects the historical philosophy of science and turns to an evolutionary one. Rather than revolutionary upheavals, Kuhn now envisions scientific progress as increasing specialization of scientific disciplines; and, in contrast to a revolutionary cycle,

* Department of Philosophy, Baylor University. Waco, TX 76798. E-Mail: james_ marcum@baylor.edu
} 
an evolutionary tree best represents Kuhn's mature philosophy of science for how science progresses.

Keywords: Evolutionary tree, Incommensurability, Kuhn, Revolutionary cycle, Symbiogenesis.

Recibido: 2019-06-08

Aceptado: 2019-09-23

\section{Introducción}

En 1962, La estructura de las revoluciones científicas de Kuhn (La estructura) se publicó como la última entrada monográfica de la Enciclopedia internacional de la ciencia unificada de Rudolf Carnap. La estructura señaló el fin de la era del positivismo/empirismo lógico en la filosofía de la ciencia (Reisch 199I). Y, a su paso, trajo una nueva visión de la ciencia. Hoy en día, la ciencia ya no es vista filosóficamente como la acumulación analítica y lógica de conocimiento. Es concebida más bien como profundamente arraigada en el tejido social y cultural de su tiempo; y así su conocimiento está configurado por ese tejido en lugar de configurarlo. Mientras los científicos investigan los fenómenos naturales, la ciencia no se cierne ya sobre las fuerzas sociales que dan forma a su vida comunitaria. No obstante, hacia el final de su carrera, Kuhn rechaza la filosofía histórica de la ciencia para reemplazarla por una filosofía evolutiva.

En este artículo, discuto brevemente qué es para Kuhn la filosofia histórica o revolucionaria de la ciencia tal como se articula en La estructura, en términos de lo que se conoce como el ciclo revolucionario de Kuhn. Luego discuto la transición de Kuhn hacia una filosofía evolutiva de la ciencia; posteriormente, exploro lo que significa esa transición en términos de su filosofía de la ciencia madura. Argumento que la filosofía de la ciencia evolutiva de Kuhn, representada por una estructura arbórea, describe mejor lo que ocurre en la práctica de la ciencia y su avance o progreso a través de esa práctica. Con este fin, recurro a un caso de la historia reciente de la biología para ilustrar la filosofía evolutiva de la ciencia de Kuhn y su estructura arbórea. Finalmente, discuto la relevancia de Kuhn para la filosofía contemporánea de la ciencia, especialmente con respecto a su énfasis en el pluralismo y el perspectivismo. Mi opinión, sin embargo, es que la mayor relevancia de Kuhn para la filosofía de la ciencia es el proveer una posible filosofía general de la ciencia. 


\section{El ciclo revolucionario kuhniano}

En La estructura, la afirmación de Kuhn según la cual "La historia sugiere que el camino hacia un firme consenso en la investigación es extraordinariamente arduo" (Kuhn 2006, p. 77; 1970, p. 15), atestigua el giro histórico que tuvo lugar alrededor de mediados del siglo XX en la filosofía de la ciencia (Bird 2005, Marcum 2015). Kuhn (2000) estuvo ciertamente a la vanguardia de ese giro que más tarde llamará 'filosofía histórica de la ciencia'. Esta filosofía comúnmente está representada por lo que los comentaristas identifican como el ciclo revolucionario de Kuhn (Bird 1999, Gregory 2013, Reingold 1980). Como se muestra en la Figura I, el ciclo comienza con el establecimiento de un paradigma para la práctica científica normal. Sin embargo, el proceso de establecer un paradigma no necesariamente es un proceso simple. Para la llamada 'ciencia pre-paradigmática', no hay consenso entre los miembros de una comunidad científica sobre los medios para investigar un fenómeno natural o sobre lo que constituye los datos aceptables para determinar qué teoría explica correctamente el fenómeno. Y así, en el mejor de los casos, el resultado es el caos y la discordia, ya que los miembros de la comunidad científica compiten para posicionar su teoría favorita en la explicación del fenómeno. Sin embargo, finalmente, una teoría capta la atención y luego la lealtad de la comunidad porque resuelve un problema crucial o un conjunto de problemas. La teoría suscita la fe de los miembros de la comunidad en el sentido de que puede guiar la investigación con respecto a otros problemas que enfrenta la comunidad (los problemas que Kuhn llama 'puzles'). Comienza de esta manera el ciclo revolucionario con la institución de la ciencia normal.

Como ningún paradigma mapea fielmente o exhaustivamente el mundo, y la historia de la ciencia ciertamente justifica esta afirmación, finalmente emergen las anomalías (Figura I). Una anomalía, para Kuhn, es una consecuencia no anticipada que se opone a la consecuencia anticipada por el paradigma. Inicialmente, una anomalía simplemente es ignorada y se atribuye a la incompetencia del investigador o se hacen modificaciones ad hoc al paradigma para explicar la observación anómala. Sin embargo, si surgen muchas anomalías y si estas anomalías ponen en duda la capacidad de la comunidad para confiar en el paradigma que guía la investigación, se proponen paradigmas alternativos y se produce la competencia entre ellos. Kuhn se refiere a la angustia mental de la comunidad durante este período de competencia como una crisis. Y, en la medida que la comunidad científica intenta resolver las anomalías, la 
práctica científica normal se convierte en una frenética práctica científica extraordinaria.

De acuerdo con Kuhn, los científicos no responden a una crisis simplemente rechazando un paradigma disfuncional. En otras palabras, los datos anómalos son insuficientes para refutar y, consecuentemente, para desechar el paradigma gobernante. ¿Por qué? Porque, según Kuhn, los paradigmas no se comparan directamente con la naturaleza, sino que con otros paradigmas y todos frente a frente a la naturaleza. Si durante la práctica de la ciencia extraordinaria un nuevo paradigma resuelve las anomalías, entonces ocurre una revolución científica y una nueva ciencia normal, inconmensurable con la ciencia normal previa, se hace cargo de guiar a la comunidad científica. La definición oficial de Kuhn para las revoluciones científicas dice que son "aquellos episodios de desarrollo no acumulativos en los que un paradigma más antiguo es sustituido en todo o en parte por otro nuevo incompatible con él" (Kuhn 2006, p. I86; cf. Kuhn I970, p. 92). Brevemente, las revoluciones científicas son cambios de paradigma. Y, después de tales cambios, los científicos trabajan en mundos nuevos o diferentes. Kuhn compara todo el proceso con un cambio de gestalt en el que el observador puede ver, por ejemplo, un pato o un conejo, pero no ambos al mismo tiempo. Con el establecimiento de una nueva ciencia normal ${ }^{\mathrm{n}}$, el ciclo revolucionario comienza de nuevo (Figura I).

\section{El árbol evolutivo kuhniano}

Kuhn comenzó a alejarse de La estructura y de su énfasis revolucionario cuando respondió a los críticos de su filosofía histórica de la ciencia y de su ciclo revolucionario. Por ejemplo, en su libro sobre la teoría del cuerpo negro y la discontinuidad cuántica, para disgusto de sus partidarios y para deleite de sus detractores (Klein y et al. 1979), Kuhn (1987) no usa el término 'paradigma' u otros términos y conceptos propios de $\mathrm{La}$ estructura. La pregunta que surge es si está comenzando a rechazar aquí la filosofía histórica de la ciencia articulada en La estructura. Dado que ahora ve el cambio científico como evolutivo en lugar de revolucionario, la respuesta es un rotundo sí (Marcum 20I5).

De hecho, incluso en las últimas páginas de La estructura, Kuhn invoca la evolución darwiniana.

El resultado neto de una sucesión de tales selecciones revolucionarias, separadas por periodos de investigación normal, es el conjunto maravillosamente adaptado de instrumentos que llamamos conocimiento científi- 
co moderno. Los sucesivos estadios de dicho proceso de desarrollo están marcados por un aumento en la articulación y especialización. Además, todo este proceso puede haberse producido, tal como suponemos ahora que ocurrió con la evolución biológica, sin recurso a una meta establecida, una verdad científica fija y permanente, de la que cada estadio del desarrollo del conocimiento científico constituya una imagen mejor. (Kuhn 2006, p. 299; cf. I970, pp. I72-I73)

En otras palabras, así como el resultado de la evolución biológica es la especiación, el resultado de la evolución científica es la especialización. Además, el resultado de la evolución científica respecto de la especialización no es la verdad por sí misma, sino más bien una mayor articulación de los fenómenos naturales, en la medida en que estos fenómenos son divididos en sus conexiones evolutivas, Sobre todo en lo que hace a la proliferación de especialidades científica.

En el discurso presidencial de 1990 a la reunión bienal de la Philosophy of Science Association, Kuhn (2000) esboza de manera preliminar una filosofía de la ciencia evolutiva. En la conferencia, Kuhn informa a la audiencia que la noción de evolución darwiniana representa mejor su imagen de la ciencia y de su progreso. Por ejemplo, después de una revolución científica siempre hay más especialidades o campos de conocimiento que antes. Kuhn analoga estas especialidades con la especiación biológica, que puede ocurrir, afirma, mediante la ramificación de una especialidad o de la unión de dos especialidades para formar una tercera. "Con el tiempo, un diagrama de la evolución de los campos, especialidades y subespecialidades científicas se parece sorprendentemente", insiste Kuhn, "a un diagrama de un árbol evolutivo biológico hecho por un lego” (Kuhn 2000, pp. 97-98).

En la Rothschild Distinguished Lecture de I99I, Kuhn (2000) rompe oficialmente con lo que él llama 'la filosofía histórica de la ciencia' y se vuelca hacia una filosofía evolutiva. Una de las principales motivaciones para el cambio de posición es el debate en el que se vio envuelto con los críticos, especialmente con respecto a la tesis de inconmensurabilidad (Marcum 20I5). Con su filosofía evolutiva de la ciencia, la tesis ya no se refiere a ninguna medida o significado no común entre paradigmas en competencia, sino a una taxonomía no común de los léxicos para especialidades científicas en evolución. En otras palabras, a medida que evoluciona una especialidad, su taxonomía léxica de los términos que refieren a objetos es sustancialmente diferente del léxico parental, sobre todo por la adición y/o eliminación de términos y conceptos. Además, el nuevo léxico mapea objetos y mundos de manera novedosa. Finalmente, 
la tesis evolutiva de la inconmensurabilidad de Kuhn sirve como un mecanismo para aislar léxicos de especialidades científicas, y para que una nueva especialidad pueda evolucionar a partir su especialidad parental.

Antes de su muerte en 1996, Kuhn estaba trabajando en un manuscrito de libro en el que estaba formulando una filosofía evolutiva de la ciencia (Hoyningen-Huene 2015, Marcum 2015). El título provisional era La pluralidad de mundos: una teoría evolutiva del desarrollo científico ( $\mathrm{La}$ pluralidad de mundos, de aquí en adelante). El libro queda inconcluso al momento de su muerte. Kuhn divide La pluralidad de mundos en tres partes, cada una compuesta de tres capítulos. En la Parte I, incluye los problemas asociados con una filosofía histórica de la ciencia, tal como se articula en La estructura; esboza también, brevemente, una visión evolutiva del desarrollo científico. Específicamente, se explaya acerca del concepto de léxico que sustituye al anterior concepto de paradigma. Un léxico representa el compendio de términos y conceptos de una especialidad científica, que los científicos usan para mapear taxonómicamente el mundo. A medida que emergen y evolucionan las especialidades científicas, aumentan los nuevos términos del léxico que mapean regiones desconocidas del mundo natural. Para los científicos de la especialidad parental, así como para los filósofos e historiadores de la ciencia, el medio requerido para comprender estos términos no en un lenguaje universal de traducción, sino más bien el bilingüismo.

En las dos partes restantes de La pluralidad de mundos, Kuhn desarrolla una filosofía de la ciencia evolutiva en términos de categorías taxonómicas no-superpuestas que se articulan dentro de léxicos en evolución. A medida que emerge una especialidad científica, se separa de la especialidad parental por la inconmensurabilidad evolutiva (Marcum 20I8). Kuhn observa, por último, que a medida que las especialidades proliferan y evolucionan, el mundo se fragmenta. Y en lugar de la visión unificada de la ciencia, concibe ahora de manera pluralista las ciencias y los mundos que ellas investigan (tal como lo muestra el título de su libro), lo que tiene implicaciones importantes por su relevancia para la filosofía de la ciencia contemporánea.

En suma, como se muestra en la Figura 2a, el árbol evolutivo general kuhniano comienza con una determinada Especialidad. Con el progreso de la especialización, particularmente cuando se encuentran fenómenos naturales que no se pueden investigar o mapear adecuadamente a través de las categorías, los términos y los conceptos léxicos de la Especialidad, se produce una división en donde emerge una nueva Especialidad $_{2}$ que termina evolucionando independientemente de la 
Especialidad. Y, a medida que el progreso científico continúa, se producen nuevas divisiones y el surgimiento y evolución de nuevas especialidades, tal como la Especialidad. En la siguiente sección se reconstruye un ejemplo de la teoría evolutiva del progreso científico de Kuhn a partir de un estudio de caso de la historia reciente de la biología.

\section{4. ¿Revolución o evolución de la biología de sistemas?}

El término 'biología' fue acuñado a principios del siglo XIX y se convirtió en la etiqueta de la disciplina que investiga los organismos vivos (Coleman 1977). Durante la primera parte del siglo XX, para disgusto de los biólogos tradicionales (Commoner I96I), una nueva biología, en contraste con la biología clásica o tradicional, aparece en el horizonte: la biología molecular con sus métodos físicos y químicos para investigar organismos (Morange 1998, Ruse 1971). Del mismo modo, a comienzos del siglo XXI aparece una nueva biología: la biología de sistemas (Trewavas 2006). De hecho, surgen varias biologías nuevas, que incluyen, por ejemplo, biología de big data, biología computacional, biología digital, biología de redes y biología sintética, por nombrar algunas. Con el objetivo de evaluar qué filosofía de la ciencia de Kuhn explica mejor los orígenes de la biología de sistemas, se reconstruye, en esta sección, estos orígenes en términos de ambas filosofías de la ciencia.

Desde principios del año 2000 la biología de sistemas se ha proclamado como un cambio de paradigma o una revolución científica kuhniana (Aderem 2005, Bothwell 2006, Marcum 2008, Trewavas 2006). "El desarrollo de tecnologías de medición de alto rendimiento para moléculas biológicas ha creado", según Kunal Aggarwal y Kelvin Lee, "un cambio de paradigma en la investigación moderna de las ciencias de la vida" (2003, p. 175). Pero surge la pregunta, en términos de la filosofía histórica de la ciencia de Kuhn, de si el surgimiento de la biología de sistemas es el producto de un genuino cambio de paradigma. En otras palabras, ¿está la biología de sistemas reemplazando otro paradigma que le es inconmensurable? ¿ $\mathrm{O}$, en lugar de ser revolucionarios, los orígenes de la biología de sistemas podrían ser evolucionarios, tal como lo afirman algunos biólogos de sistemas (Kellenberger 2004, Westerhoff y Palsson 2004)? En esta sección se exploran ambas preguntas.

La cuestión de si el surgimiento de la biología de sistemas representa un cambio de paradigma o una revolución científica kuhniana, puede abordarse en términos del ciclo revolucionario de Kuhn (Figura I). Como se señaló anteriormente, el enfoque predominante para la investigación 
biológica, durante la mayor parte del siglo XX, fue molecular. Los biólogos moleculares afirman que al reducir los organismos a sus macromoléculas, particularmente a los genes y las proteínas, los procesos de la vida pueden explicarse exhaustivamente por mecanismos moleculares. De hecho, el Proyecto del Genoma Humano tiene que como guía el supuesto de que si el genoma se puede secuenciar en términos de su composición de ADN, no solo se puede explicar sino que también manipular la vida y muchas enfermedades crónicas, como el cáncer, pueden ser curadas (Wheeler y Wang 2013). En otras palabras, los organismos vivos son su ADN, la verdadera fuente de la vida (Crick 198I). El enfoque molecular en biología sigue desempeñando un papel paradigmático para muchos biólogos.

Ya a fines del siglo XX, Richard Strohman (1997) presagia la próxima revolución kuhniana en biología. La razón de su pronóstico es que las diferencias entre las especies a nivel fenotípico no son reducibles a diferencias a nivel genotípico -la denominada 'brecha de genotipo-fenotipo' (Strohman 2002). En otras palabras, el presupuesto del determinismo genético comienza a experimentar problemas o anomalías (Auletta 20I0, Hopkin 2009, Portin 2015). Una de las principales anomalías que cita Strohman (1997) es el alto grado de similitud genética entre especies, por ejemplo, el $98 \%$ de similitud entre la secuencia de ADN humano y chimpancé. La brecha se está abordando actualmente a través de la epigenética, que concierne a la regulación de la expresión génica por elementos que no son $\mathrm{ADN}$ pero que también pueden heredarse tanto mitótica como meióticamente (Deans y Maggert 2015). La epigenética también es defendida como un cambio de paradigma o una revolución científica kuhniana (Baluška y Witzany 2013, Vineis 20I0). Como escribe Nessa Carey, frente a la revolución epigenética, la comunidad biológica está en "medio de un cambio de paradigma" (2012, p. I70).

Una de las consecuencias de la revolución epigenética es la redefinición del gen qua unidad de la herencia (Griffiths y Stotz 2006, Hopkin 2009, Portin 2002). Según el paradigma de la biología molecular, el gen es una secuencia única de ADN que codifica para una proteína, es decir, son genes codificadores de proteínas. Pero, como lo ha demostrado la investigación reciente, el gen qua unidad de la herencia no tiene que ser solo ADN que codifica una proteína; también puede ser ADN que no codifica proteínas en términos de pequeños ARNs (ARN mensajero, de transferencia o ribosómico), pero que están involucrados en la regulación génica y la herencia epigenética transgeneracional (He y Hannon 2004, Heard y Martienssen 2014). Aunque están disponibles en la literatura varias definiciones para el gen posgenómico, Petter Portin y Adam 
Wilkins proporcionan una definición bastante útil para ilustrar cuán compleja se está volviendo la noción del gen.

Un gen es una secuencia de ADN (cuyos segmentos componentes no necesariamente tienen que ser físicamente contiguos) que especifica una o más secuencias relacionadas de ARNs/proteínas que a la vez son suscitadas por las RRGs [redes reguladoras genéticas] y que participan como elementos en los RRGs, a menudo con efectos indirectos, o como productos de RRGs, siendo estos últimos los que producen los efectos fenotípicos más directos (20I7, pp. I36I-I362).

Aunque los autores reconocen que la definición es engorrosa y que enfatiza lo molecular, argumentan que limitar el gen a productos moleculares la hace más precisa que las definiciones que incluyen solo atributos fenotípicos. Pero entonces la brecha sigue presente.

Otro problema o posible anomalía para la biología molecular han sido las cantidades masivas de datos ómicos moleculares que genera pero que no pueden comprenderse (Marx 20I3, Sabina 20I9). Aunque la bioinformática ayuda a gestionar los datos, tampoco puede darles sentido (Greene et al. 20I4). En respuesta a estos datos, la biología de sistemas ha surgido como un nuevo paradigma, en un intento por parte de los biólogos de dar sentido o interpretar estos datos (Kitano 2002). Finalmente, en una revisión de los recientes desafíos al paradigma mendeliano que surge en la genética contemporánea, Portin concluye que "todo el nuevo paradigma emergente de la genética parece ser una parte integral del nuevo paradigma propuesto para biología, el paradigma de la Biología de Sistemas" ( 20I5, p. 24). De hecho, el paradigma de la biología de sistemas sirve no solo para revolucionar la genética sino también otras ramas de la biología, desde la biología celular hasta la ecología y la biología evolutiva. Por lo tanto, los biólogos ahora viven en "una era de sistemas" (Trewavas 2006, p. 2420), en oposición a una previa "era de las máquinas" (Elsassar I998, p. xxvi).

Sin embargo, en términos del ciclo revolucionario kuhniano, surgen varias preguntas acerca de la relevancia del ciclo para el surgimiento de la biología de sistemas. ¿Las anomalías anteriores son realmente anomalías en el sentido de que van en contra del paradigma de la biología molecular? La respuesta no es tan clara. Por ejemplo, muchos fenómenos epigenéticos se conocían antes del establecimiento de la biología molecular como paradigma y, por lo tanto, no son exactamente anomalías en términos de la comprensión de Kuhn de las anomalías, es decir, no surgieron en contra de las expectativas de la biología molecular. Además, ¿estas su- 
puestas anomalías condujeron a una crisis dentro de la comunidad de la biología molecular? La respuesta es, en cierto sentido, negativa: aunque practicaron una ciencia extraordinaria kuhniana afín a la biología de sistemas, los biólogos no abandonaron la ciencia normal asociada con la biología molecular.

Ciertamente, la biología de sistemas desarrolla los modelos matemáticos y las simulaciones computacionales necesarias para dar sentido a los datos generados a través de la biología molecular; pero, ¿es este un cambio kuhniano de paradigma? En otras palabras, ¿la biología de sistemas reemplazó a la biología molecular de tal manera que los biólogos ya no la practicaron más? Una forma de responder a esta pregunta es mirar el número de publicaciones anuales en PubMed (https://www. ncbi.nlm.nih.gov/pubmed/) utilizando 'biología molecular' o 'biología de sistemas' como términos de búsqueda. Como se muestra en la Figura 3, el número de publicaciones anuales en biología de sistemas ciertamente ha aumentado constantemente desde comienzos del siglo XXI, pero también lo ha hecho la de biología molecular. De hecho, en 2018 hay casi cuatro veces más publicaciones en biología molecular que en biología de sistemas. La biología molecular sigue viva y sana y es muy exagerada la noticia de su muerte (Rheinberger 2007).

¿Puede la filosofía evolutiva de la ciencia de Kuhn venir al rescate? Como se muestra en la Figura 2b, la aparición de la biología de sistemas encaja perfectamente en el árbol evolutivo general kuhniano (Figura 2a). Comenzando con la biología clásica y sus limitaciones con respecto a las descripciones morfológicas y las clasificaciones taxonómicas, el surgimiento de la biología molecular permitió a los biólogos explicar las funciones biológicas en términos de mecanismos moleculares. Pero, en cierto sentido, la biología molecular fue víctima de su propio éxito al generar demasiados datos moleculares a partir de tecnologías ómicas. El diluvio de datos no es una anomalía en la medida que abrió un nicho epistémico para que lo ocupe una especialidad como la biología de sistemas. Y lo hace con éxito, como lo demuestra el continuo aumento anual de las publicaciones (Figura 3). Sin embargo, el árbol evolutivo kuhniano, en lo que respecta a la división de linajes, si bien proporciona un medio para comprender o visualizar el surgimiento de la biología de sistemas, no explica el mecanismo real de la evolución de la biología de sistemas a partir de la biología molecular.

Como Kuhn señaló en el discurso presidencial ante la reunión de la Philosophy of Science Association de 1990, una especialidad científica puede surgir de dos maneras: (I) separándose de una especialidad madre o 
(2) uniendo dos especialidades para formar una nueva. El último medio, en el que dos especies se unen para formar una nueva, se conoce como 'simbiogénesis' (Margulis 1998). Por ejemplo, las células eucariotas evolucionaron a partir de la simbiosis entre bacterias y arqueas. Del mismo modo, una nueva especialidad científica puede surgir de la simbiosis de dos (o más) especialidades. Como se muestra en la Figura 4, la biología de sistemas es el resultado de la simbiosis de la biología molecular con la teoría de sistemas. La teoría de sistemas se atribuye comúnmente a Ludwig von Bertalanffy (1974), quien define un sistema como un conjunto interrelacionado de componentes regidos por un conjunto de principios generales de sistemas. Por lo tanto, la teoría de sistemas proporciona los medios conceptuales y computacionales para dar sentido a los datos generados por la biología molecular. Finalmente, debe señalarse que aunque la biología molecular y la teoría de sistemas se unen para formar la biología de sistemas, las antiguas especialidades continúan existiendo de manera independiente, al igual que las bacterias y las arqueas continúan existiendo de forma independiente después de unirse para formar células eucariotas.

En cierto sentido, la filosofía histórica de la ciencia de Kuhn es demasiado revolucionaria o artificial y falla en la representación del crecimiento orgánico de la ciencia y de su desarrollo. La filosofía evolutiva de la ciencia de Kuhn, por otro lado, proporciona un medio para reconstruir la narrativa orgánica y de desarrollo de la biología de sistemas. En lugar de revolucionaria, esa narrativa se reconstruye mejor como evolutiva, denota así con mayor precisión la relación entre la biología de sistemas y las otras ciencias biológicas, especialmente la biología molecular. En lugar de un cambio revolucionario o un cambio de paradigma, la biología de sistemas representa una escisión evolutiva a partir de la biología molecular a través de la unión simbiótica de la biología molecular y la teoría de sistemas (Figura 4). En resumen, la biología de sistemas representa una nueva especialidad en las ciencias biológicas que tiene a la biología molecular como su predecesora. Ambas habitan el mismo mundo pero en nichos diferentes.

\section{Kuhn y la filosofía contemporánea de la ciencia}

¿Cuál es la relevancia de la filosofía evolutiva de la ciencia de Kuhn para la filosofía de la ciencia contemporánea? Como opina un comentador: «La mayoría de los filósofos que han elogiado o desafiado la opinión de Kuhn se han centrado casi exclusivamente en la ERC [La estructura 
de las revoluciones científicas] ... [y] no han prestado suficiente atención a las obras posteriores a la ERC de Kuhn, casi como si no hubiese tenido nada relevante que decir o simplemente nada que decir después de ERC" (Politi 20I8, pp. 2267-2268). Ciertamente esto es lamentable por al menos dos razones. Primero, Kuhn, como se señaló anteriormente, rechaza la filosofía histórica de la ciencia articulada en La estructura en la medida en que reemplaza el concepto de paradigma con el concepto de léxico. En segundo lugar, para el avance científico, adopta una filosofía evolutiva de la ciencia con énfasis en la especialización en lugar de la revolución o el cambio de paradigma.

En esta sección, discuto brevemente primero la relevancia de la filosofía de la ciencia evolutiva de Kuhn respecto al énfasis de la filosofía contemporánea de la ciencia en el pluralismo y el perspectivismo. Según el pluralismo, ninguna ciencia domina a las otras en cuanto a establecer un programa para determinar qué constituye una ciencia (Kellert et al. 2006). Atrás quedaron los días en que la física, con su positivismo lógico y empirismo, intimidaba a otras ciencias como la química y la biología, a tal punto de obligarlas a cumplir o ajustarse a su modelo analítico. En la filosofía de la ciencia actual cada ciencia se estudia por derecho propio y bajo sus propios términos. La filosofía de la ciencia de Kuhn, tal como se articula en La pluralidad de mundos, es relevante para el énfasis pluralista de la filosofía de la ciencia contemporánea: las especialidades científicas surgen y evolucionan junto con sus respectivas filosofías, abarcando progresivamente los mundos que están abiertos tanto a la investigación científica como al análisis filosófico. Según el perspectivismo, no hay una posición única o privilegiada desde la cual ver un objeto (Giere 2006). La relevancia de Kuhn reside aquí en que la perspectiva científica y filosófica es evolutiva en el sentido de que la ciencia, junto con su filosofía, se están desplegando de manera orgánica y dinámica. En otras palabras, la ciencia progresa en la medida que aumenta el número de especialidades y a medida que se abren nuevos nichos a través de la interacción de estas especialidades. La filosofía de la ciencia hace lo mismo en cuanto a la proliferación de sus especialidades. Lo singular aquí es que las ciencias y sus filosofías están integradas en una pluralidad de mundos en evolución. La visión epistémica es entonces orgánica y dinámica: el observador y lo observado son parte del proceso evolutivo.

Sin embargo, la mayor relevancia de Kuhn para la filosofía de la ciencia contemporánea radica en su filosofía general de la ciencia que, a primera vista, parece contradictoria con la discusión anterior sobre el pluralismo y el perspectivismo. Recientemente, Stathis Psillos (2012, 
2016) ha propuesto y defendido una filosofía general de la ciencia. $\mathrm{Cu}$ riosamente, Psillos afirma que las diversas ciencias son análogas a las especies biológicas en el sentido de que cada una, ya sea una especie o una determinada ciencia, tiene una historia particular o "esencia histórica" (20I2, p. IOO). Aunque no desarrolla mayormente esta analogía, la filosofía evolutiva de la ciencia de Kuhn proporciona un medio para rastrear las raíces o esencias históricas de las diversas ciencias hasta su ancestro común y, por esto, constituye una posible candidata para una filosofía general de la ciencia. Como se muestra en la Figura 5, la filosofía evolutiva de la ciencia de Kuhn puede servir como una filosofía general de la ciencia en la medida que mapea las relaciones de las diversas ciencias que evolucionan. La Ciencia, por ejemplo, podría representar una ciencia aristotélica, que según Psillos exhibe dos características compartidas por otras ciencias qua ciencia general. La ciencia en general tiene "una especial demanda de conocimiento y una relación especial con la realidad" (20I2, p. IOI). La Ciencia ${ }_{3}$ comparte entonces estos rasgos con la Ciencia y la Ciencia, dada su ascendencia común, pero la Ciencia también exhibe características únicas específicas. Por ejemplo, la biología hace enunciados epistémicos sobre el mundo biológico como lo hace la física sobre el mundo físico y la química sobre el mundo químico. Estos enunciados no requieren ser legaliformes para ser explicativos (Brandon 1997).

Psillos (2016) identifica cuatro condiciones o dimensiones de una filosofía general de la ciencia que la filosofía evolutiva de la ciencia de Kuhn satisface o exhibe. La primera es epistémica en lo que respecta a ofrecer una explicación del descubrimiento y la justificación del conocimiento científico. La filosofía evolutiva de la ciencia de Kuhn satisface esta condición en relación a la naturaleza evolutiva de la formación y validación de teorías. Hay un "ajuste epistémico" entre la investigación científica de la realidad y el descubrimiento que luego respalda su conocimiento del mundo. La siguiente condición es metafísica: la ciencia se esfuerza por identificar las categorías ontológicas de la realidad. Nuevamente, la filosofía evolutiva de la ciencia de Kuhn cumple con esta condición, ya que a medida que la ciencia evoluciona multiplicando especialidades, revela las categorías ontológicas que constituyen el mundo. La tercera condición es conceptual respecto a los contextos teóricos y experimentales en los que la ciencia enmarca la realidad. La filosofía evolutiva de la ciencia de Kuhn satisface esta condición en términos de la evolución cooperativa de los enfoques teóricos y experimentales para investigar el mundo. La condición final es práctica: tiene que ver con las 
dimensiones éticas, sociales y políticas de la ciencia. La filosofía evolutiva de la ciencia de Kuhn cumple con esta condición con respecto a la naturaleza adaptativa de la ciencia, a medida que evoluciona para dar cuenta de la realidad ética, social y política. En resumen, la filosofía evolutiva de la ciencia de Kuhn satisface las condiciones de Psillos para una filosofía general de la ciencia.

Finalmente, aunque la filosofía evolutiva de la ciencia de Kuhn representa una posible candidata para restablecer una filosofía general de la ciencia, cumple esta tarea no en el sentido tradicional de proponer una imagen de ciencia en la cual todas las ciencias particulares deban encajar (Marcum 20I7, 20I8). Más bien, la imagen evolutiva de la ciencia de Kuhn promueve una imagen general alternativa respecto de las relaciones históricas de las diversas ciencias naturales y sus especialidades a medida que ellas evolucionan (compare las Figuras 2 y 5). De esta manera explica tanto el énfasis pluralista como perspectivista de la filosofía de la ciencia contemporánea al dar cuenta de las relaciones evolutivas entre las especialidades científicas en términos de sus ancestros comunes. Su objetivo no es forzar, en consideración a sus características fundamentales, a las diversas especialidades a un solo molde (la física, por ejemplo). Más bien, su objetivo es dar cuenta de cómo estas especialidades avanzan y progresan como un sistema integrado en el que las diversas ciencias interactúan y emergen unas de otras, por ejemplo, por simbiosis evolutiva.

\section{Conclusión}

En suma, la filosofía de la ciencia evolutiva de Kuhn ofrece la posibilidad de una filosofía de la ciencia general fecunda que aborde la naturaleza evolutiva de la ciencia en términos de la proliferación de especialidades. Para Kuhn, la especialización científica es crucial para investigar y comprender la pluralidad de mundos, no en términos de hechos objetivos $\mathrm{y}$ verdades universales, sino con respecto a los componentes y facetas específicas que los constituyen, es decir, en relación a la creciente articulación de esos mundos, al ser representados con mayor cercanía y precisión respecto de sus coyunturas evolutivas. En otras palabras, la ciencia avanza a través de la evolución de especialidades que capturan estos mundos cada vez con mayor detalle. Como lo demuestra el estudio del caso histórico de la biología de sistemas, la imagen evolutiva de la ciencia de Kuhn no solo explica el énfasis pluralista y perspectivista de la filosofía contemporánea, sino que también ofrece un tipo de consenso 
entre las ciencias no en términos de reducirlas a una sola ciencia, sino más bien con respecto a trazar las relaciones conceptuales entre ellas a medida que evolucionan a partir de ancestros comunes*.

Figura I. El ciclo revolucionario kuhniano (ver el texto para los detalles).

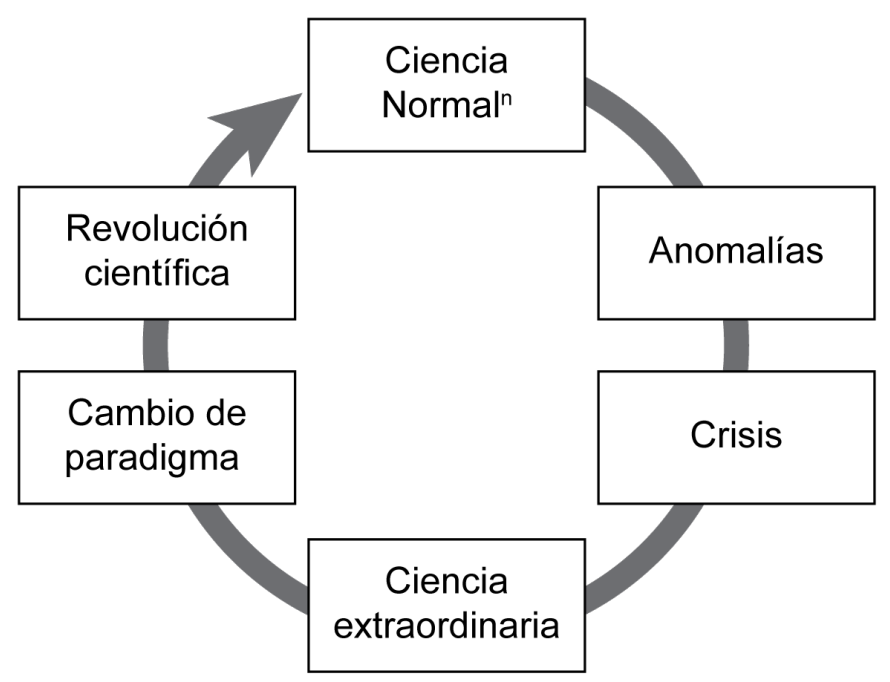

Figura 2. El árbol evolutivo kuhniano (ver el texto para los detalles).

(a) Árbol evolutivo general

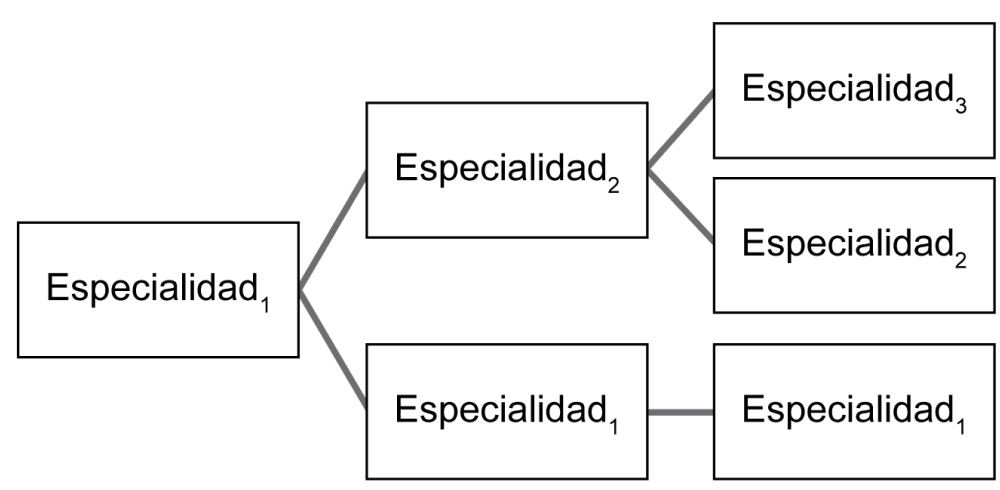


(b) Árbol evolutivo para la biología de sistemas

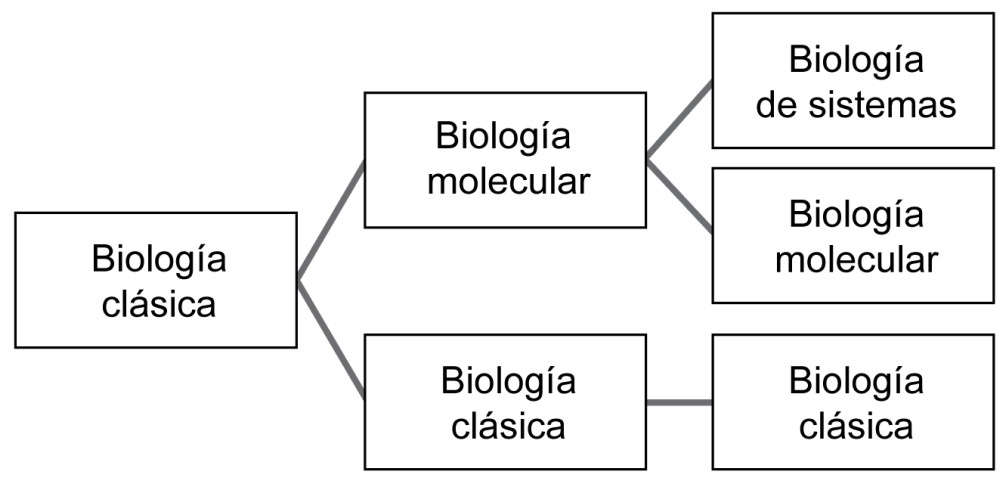

Figura 3. Publicaciones anuales en PubMed para "biología molecular" y "biología de sistemas" (ver el texto para los detalles).

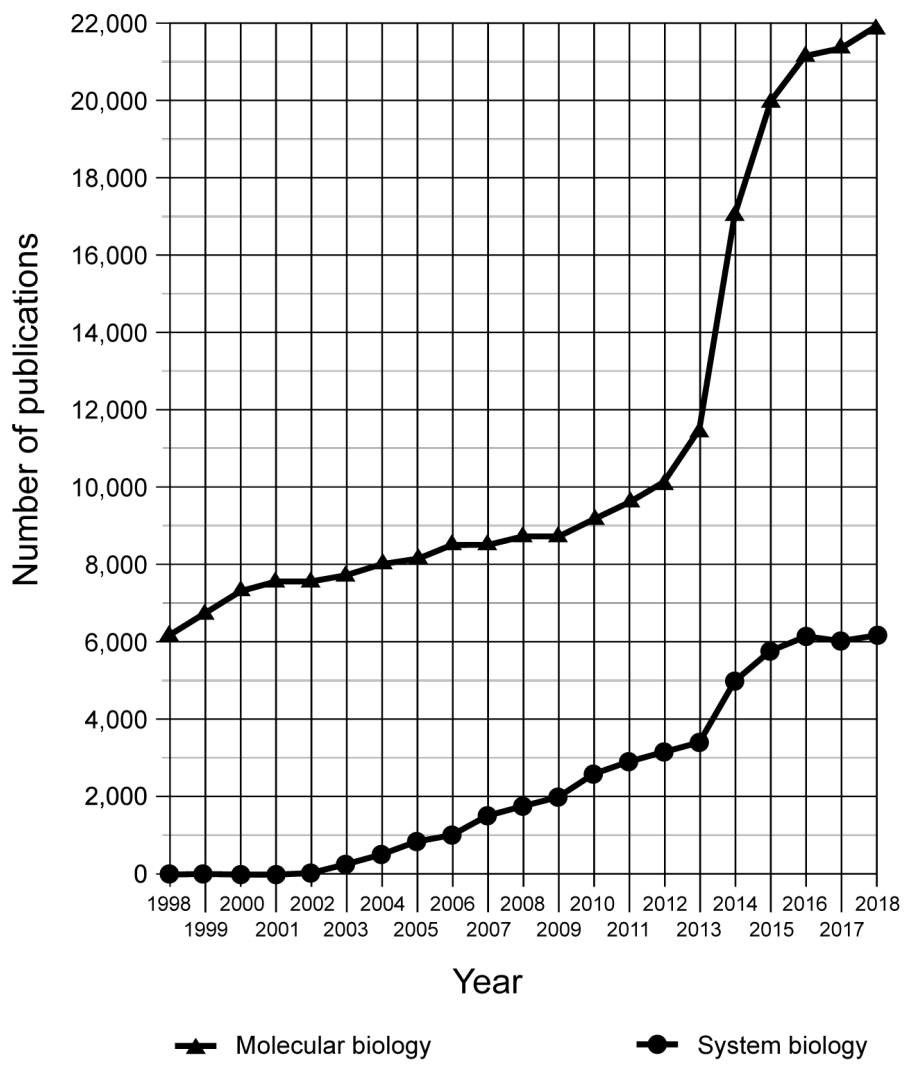


Figura 4. Simbiogénesis de la biología de sistemas (ver el texto para los detalles).

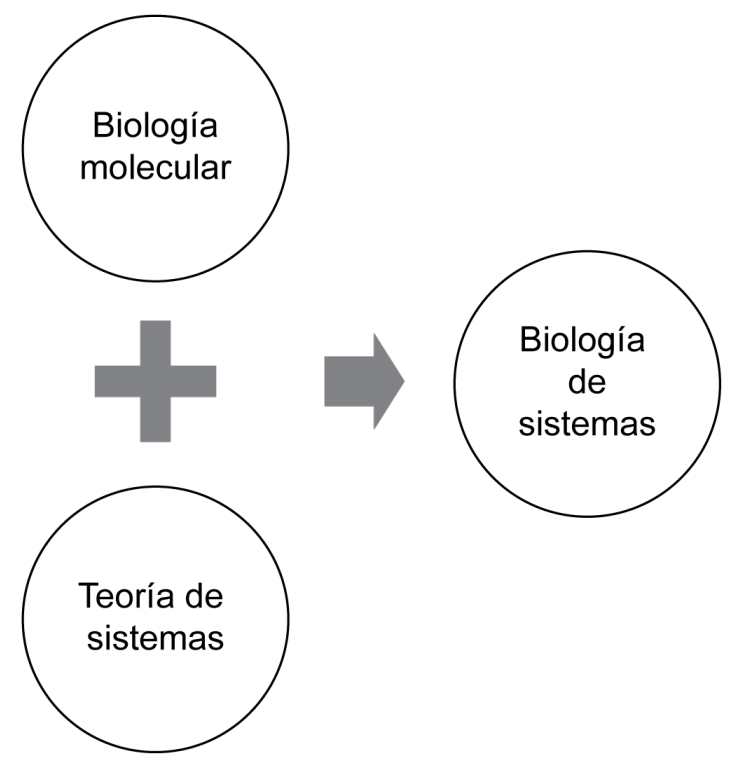

Figure 5. La filosofía de la ciencia evolutiva de Kuhn como filosofía de la ciencia general (ver el texto para los detalles).

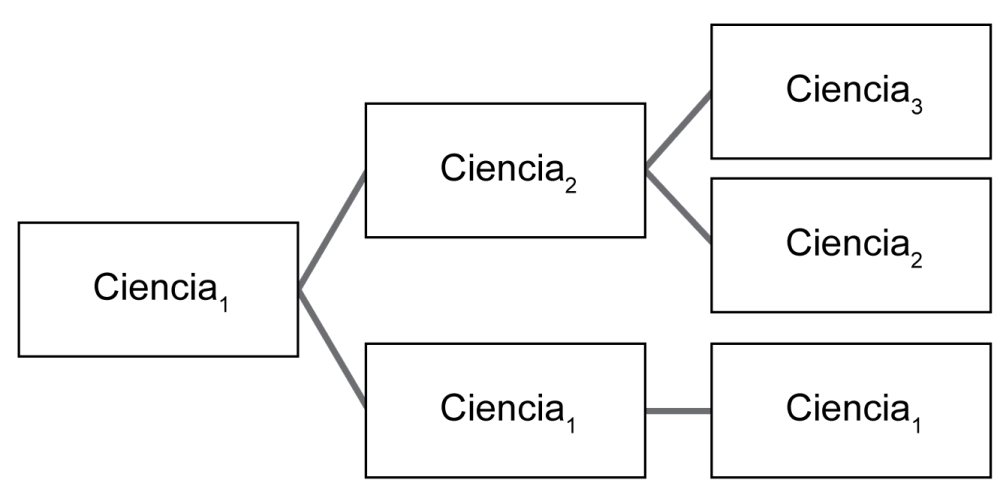

* Traducción de Julio Torres Meléndez, Pablo Melogno y Rubén Aguilar. 


\section{Referencias bibliográficas}

Aderem, A. (2005). "Systems Biology: Its Practice and Challenges". Cell I2: $5 \mathrm{II}-5 \mathrm{I3}$.

Aggarwal, K., and Lee, H.K. (2003). "Functional Genomics and Proteomics as a Foundation for Systems Biology. Briefings in Functional Genomics 2: I75-I84.

Auletta, G. (2010). “A paradigm Shift in Biology?" Information I: 28-59.

Baluška, F., and Witzany, G. (2013). "At the Dawn of a New Revolution in Life Sciences". World Journal of Biological Chemistry 4: I3-I5.

Bertalanffy, L. von (1974). General systems theory: Foundations, development, applications, revised edition. New York: Braziller.

Bird, A. (1999). "Scientific Revolutions and Inference to the Best Explanation". Danish Yearbook of Philosophy 34: 25-42.

- (2005). "Naturalizing Kuhn". Proceedings of the Aristotelian Society I05: 99-II7.

Brandon, R.N. (1997). "Does Biology have Laws? The Experimental Evidence". Philosophy of Science 64: S444-S457.

Carey, N. (2012). The epigenetics revolution. How modern biology is rewriting our understanding of genetics, disease, and inheritance. New York: Columbia University Press.

Coleman, W. (1977). Biology in the Nineteenth Century: Problems of form, function, and transformation. Cambridge: Cambridge University Press.

Commoner, B. (196I). "In Defense of Biology". Science 133: I745-I748.

Crick, F. (198I). Life itself: Its origin and nature. New York: Simon and Schuster.

Deans, C., and Maggert, K.A. (2105). "What do You Mean, "Epigenetic"?" Genetics I99: 887-896.

Elsassar, W.M. (1998). Reflections on a theory of organisms: Holism in biology. Baltimore, MD: The Johns Hopkins University Press.

Giere, R.N. (2006). Scientific perspectivism. Chicago: University of Chicago Press.

Greene, C.S., Tan, J., Ung, M., Moore, J.H., and Cheng, C. (20I4). "Big Data Bioinformatics”. Journal of Cellular Physiology 229: I896-I90o.

Gregory, A. (2013). "Kuhn and Taxonomies of History". Philosophy Study 3: 412-430.

Griffiths, P.E., and Stotz, K. (2006). "Genes in the Postgenomic Era". Theoretical Medicine and Bioethics 27(6), 27: 499-52I. 
He, L., and Hannon, G.J. (2004). "MicroRNAs: Small RNAs with a Big Role in Gene Regulation”. Nature Reviews Genetics 5: 522-53I.

Heard, E., and Martienssen, R.A. (20I4). "Transgenerational Epigenetic Inheritance: Myths and Mechanisms". Cell I57: 95-I09.

Hopkin, K. (2009). "The Evolving Definition of a Gene". BioScience 59: 928-93I.

Hoyningen-Huene, P. (2015). "Kuhn's Development Before and After Structure". In W.J. Devlin

and A. Bokulich (eds.). Kuhn's Structure of Scientific Revolutions-5o Years On, I85-195. New York: Springer.

Kellenberger, E. (2004). "The Evolution of Molecular Biology". EMBO Reports 5: 546-549.

Kellert, S.H., Longino, H.E., Waters, C.K. (eds.) (2006). Scientific pluralism. Minneapolis, MN: University of Minnesota Press.

Kitano, H. (2002). "Systems Biology: A Brief Overview". Science 295: I662I664.

Klein, M.J., Shimony, A., and Pinch, T.J. (1979). "Paradigm Lost? A Review Symposium". ISIS 70: 429-440.

Kuhn, T.S. (1970). The structure of scientific revolutions, 2nd edition. Chicago: University of Chicago Press. Traducción al español de Carlos Solis, Kuhn, T.S. (2006). La estructura de las revoluciones cientícas. México: F.C.E.

(1977). The essential tension: Selected studies in scientific tradition and change. Chicago: University of Chicago Press.

(1987). Black-body theory and the quantum discontinuity, I8941912, 2nd edition. Chicago: University of Chicago Press.

(2000). The road since 'Structure': Philosophical essays, I970-1993, with an autobiographical interview. Chicago: University of Chicago Press.

Marcum, J.A. (2008). "Does Systems Biology Represent a Kuhnian Paradigm Shift?" New Phytologist I79: 587-589.

- (2015). Thomas Kuhn's revolutions: A historical and an evolutionary philosophy of science? London: Bloomsbury Publishing. (2017). "Evolutionary Philosophy of Science: A New Image of Science and Stance towards General Philosophy of Science". Philosophies 2: I-I2.

(2018). "Revolution or Evolution in Science? A Role for the Incommensurability Thesis?" In M. Mizahi (ed.), The Kuhnian Image of Science: Time for a Decisive Transformation?, 155-I73. London: Rowman \& Littlefield. 
Margulis, L. (1998). Symbiotic planet: A new look at evolution. New York: Basic Books.

Marx, V. (2013). "Biology: The Big Challenges of Big Data". Nature 498: 255-260.

Morange, M. (1998). A history of molecular biology. Cambridge, MA: Harvard University Press.

Politi, V. (20I8). "Scientific Revolutions, Specialization and the Discovery of the Structure of DNA: Toward a New Picture of the Development of the Sciences". Synthese 195: 2267-2293.

Portin, P. (2002). "Historical Development of the Concept of the Gene". Journal of Medicine and Philosophy 27: 257-286.

(2015). "The Development of Genetics in the Light of Thomas Kuhn's Theory of Scientific Revolutions". Recent Advances in DNA E Gene Sequences 9: 14-25.

and Wilkins, A. (2017). "The Evolving Definition of the Term 'Gene'”. Genetics 205: 1353-1364.

Psillos, S. (2012). "What is General Philosophy of Science?" Journal for General Philosophy of Science 43: 93-I03.

(2016). "Having Science in View: General Philosophy of Science and Its Significance”. In P. Humphrey (ed.), The Oxford Handbook of Philosophy of Science, I37-I62. New York: Oxford University Press.

Reingold, N. (1980). "Through Paradigm-Land to a Normal History of Science". Social Studies of Science IO: 475-496.

Reisch, G.A. (199I). "Did Kuhn Kill Logical Empiricism?" Philosophy of Science 58: 264-277.

Rheinberger, H.J. (2008). "What Happened to Molecular Biology?" BioSocieties 3: 303-310.

Ruse, M.E. (197I). "Reduction, Replacement, and Molecular Biology". Dialectica 25: 39-72.

Sabina, L. (2019). "The Challenges of Big Data Biology". eLife 8: DOI:I0.7554/eLife.4738I.

Strohman, R.C. (1997). "The Coming Kuhnian Revolution in Biology". Nature Biotechnology 15: 194-200.

- (2002). "Maneuvering in the Complex Path from Genotype to Phenotype". Science 296: 70I-703.

Trewavas, A. (2006). "A Brief History of Systems Biology". Plant Cell I8: 2420-2430.

Vineis, P. (2010). "The Research Program in Epigenetics: The Birth of a New Paradigm". In A.H. Haslberger and S. Gressler (eds.), Epigenetics 
and Human Health: Linking Hereditary, Environmental and Nutritional Aspects, I-6. Weinheim: Wiley-VCH.

Westerhoff, H.V., and Palsson, B.O. (2004). "The Evolution of Molecular Biology into Systems Biology". Nature Biotechnology 22: I249-I252.

Wheeler, D.A., and Wang, L. (20I3). "From Human Genome to Cancer Genome: The First Decade". Genome Research 23: I054-I062. 